\title{
AUTOMOTIVE VARIABLE ENGINE VALVE LIFT MECHANISM CONTROLLED BY A HYDRAULIC 3-STEP ROTARY ACTUATOR
}

\author{
Yasukazu SATO*, Yukinori NISHIMOTO**, Yoshitomo FUKUSHIMA** \\ and Takuya NAGATAKI** \\ * Department of Mechanical Engineering, Faculty of Engineering \\ Yokohama National University \\ 79-5, Tokiwadai, Hodogayaku, Yokohama, Kanagawa, 240-8501 Japan \\ (E-mail: yasukazu@ynu.ac.jp) \\ ** Research \& Development Center, MIKUNI CORPORATION \\ 2480, Kuno, Odawara, Kanagawa, 250-0055 Japan
}

\begin{abstract}
Variable valve lift (VVL) system for automotive engines is one of the key technologies to attain improvement of fuel economy and power output and reduction of emission. This paper presents a highly reliable VVL mechanism controlled by a hydraulic 3-step rotary actuator. 3-step VVL with high-, middle- and low-valve lift, is realized by the pivot shifting of an intermediate cam placed between a camshaft and valve tappet. The hydraulic actuator with no electric device is installed in a cylinder head and its rotary positioning generates 3-step rotation of a specially-designed sprag cam. The actuator can work in low supply pressure of $0.1 \mathrm{MPa}$ and control 3-step angles within the interval in which the valve is at rest during camshaft rotation. This paper describes the analytical simulation model of the VVL system, and the experimental evaluation for the developed VVL mechanism and hydraulic 3-step rotary actuator.
\end{abstract}

\section{KEY WORDS}

Hydraulic Rotary Actuator, Variable Valve Lift, Engine Valve Actuation, Cam Mechanism

\section{INTRODUCTION}

Variable valve actuation (VVA) is one of the remarkable technologies to attain improvement of fuel economy and power output and reduction of emission, all over the engine rotational speed range. ${ }^{(1)-(5)}$ VVA systems of various mechanisms have been thrown into the market from the second half of the 1980s and have been adopted with many automobile manufacturers' engine. VVA allows some or all of the timing, lift and duration of the intake or exhaust valves, or both, to be changed while the engine is in operation. According to the controlled object, VVA is roughly divided into three types; variable valve timing (VVT) varying the phase of valve actuation, variable valve lift (VVL) varying the stroke of the valve, and variable valve event (VVE) varying the duration in which the valve is opening. Although a completely step-less VVA in all the controlled objects is an ideal, even if technically possible, the development of a practical system is worthwhile, which employs one or some features of VVA system efficiently from viewpoints of cost restriction, durability and maintainability.

In this paper, the authors aimed at the development of the simple VVA system, and paid their attention to the VVL mechanism with 3-step lift patterns.

Practical implementation conditions of the VVL mechanism into an engine are settled as follows;

1) Since its fluid power source is an engine oil pump already equipped on an engine, it can operate with the engine oil of the minimum operating pressure of about 0.1-0.2MPa.

2) It has high rigidity for holding valve lift. 
3) It can control 3-step lift without any electric sensor.

4) It has satisfying response within $0.3 \mathrm{~s}$ in the valve lift change as the VVL system for an engine of passenger car.

The VVL mechanism driven by a hydraulic rotary actuator is developed for above purposes. This paper presents the evaluation of its performance by both the simulation and experimental approach.

\section{VVL MECHANISM CONTROLLED BY A HYDRAULIC 3-STEP ROTARY ACTUATOR}

\section{Concept of VVL Mechanism with a Shifting Pivot of Intermediate Cam}

In the developed VVL mechanism, the valve lift is varied by pivot shift of an intermediate cam placed between a camshaft and valve tappet. Fig.1 shows the principle of the VVL. As the pivot of the intermediate cam moves away from the camshaft, the valve lift becomes shortened. Although Fig.1 shows 6-step VVL characteristics as one example, the developed VVL system was designed to generate 3-step of high-, middle- and low- valve lift which were frequently used in the actual VVL control. Therefore, 3-step positioning of the pivot of the intermediate cam is the key of the developed VVL system.

Combination of a Sprag Cam and Hydraulic Rotary Actuator

In the duration in which the valve is opening due to the camshaft in action, large force is generated at the pivot of the intermediate cam as the reaction force of the camshaft pushing the valve tappet. A component of the reaction force perpendicular to the direction of the pivot shift is borne by the slide guide of the intermediate cam retainer, and a component to the direction of the pivot shift works as a load to the VVL mechanism. From the viewpoint of installing the VVL mechanism into the small and restricted place around the cylinder head, it is unpractical to adopt an actuator which always generates force overcoming this load, because such an actuator is generally large in its body size and needs large power input.

The developed VVL mechanism is characterized by combining the sprag cam with specially designed cam profile, and a hydraulic rotary actuator. For the duration of the valve opening, large reaction force at the pivot of intermediate cam is borne by the sprag cam. Then, the position of the pivot is held with high stiffness. For the duration in which the valve is closing due to the camshaft at rest, the pivot position is controllable by the drive force generated at the hydraulic rotary actuator, because only small initial load acts on the pivot.

Thus, the developed VVL system can keep the pivot position by the sprag cam for the duration of the valve opening, and can operate it by a small hydraulic actuator and low pressure source such an engine oil pump except for that duration.

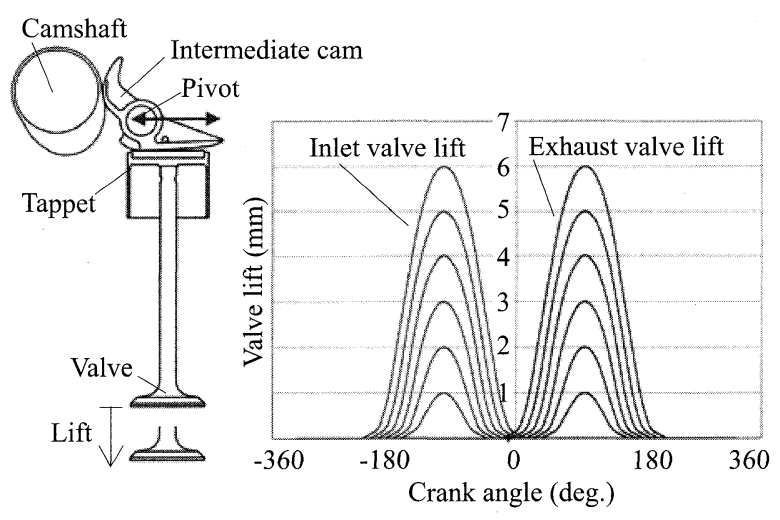

Figure1 Principle of variable valve lift with pivot shifting

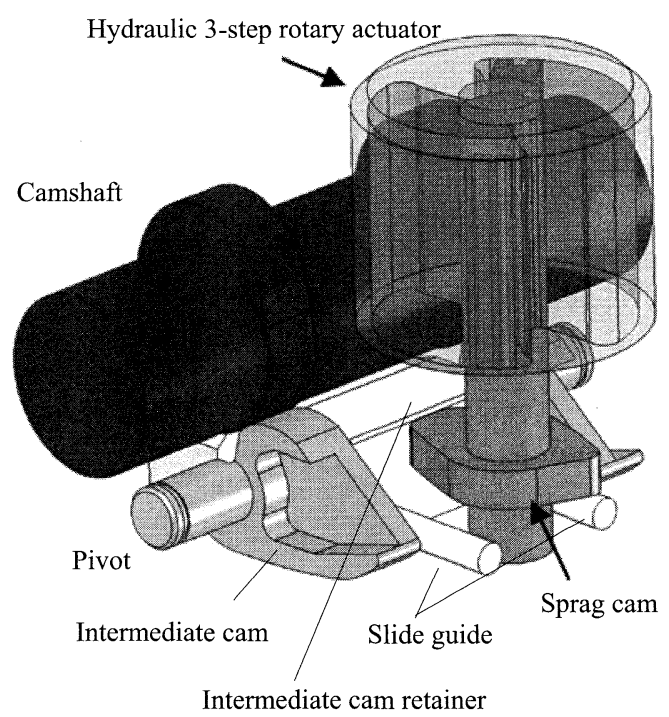

Figure 2 VVL mechanism controlled by the hydraulic 3-step rotary actuator

VVL Mechanism and Hydraulic Rotary Actuator

The schematic view of the VVL mechanism is shown in Fig.2. The hydraulic rotary actuator generates 3-step swing rotation by control of the oil pressure in the chambers inside. The pivot position of the intermediate cam shifts by the rotation of the sprag cam driven by the rotary actuator. The body of the rotary actuator is designed small so that the VVL mechanism could be added on the cylinder head of a conventional engine without complicated arrangement, and be placed between the intake or exhaust valves on each cylinder. Fig. 3 shows the relation between the swing angle of the rotary actuator, the pivot position of the intermediate cam and the valve lift.

The cam profile of the sprag is designed to stabilize its rotation angle by the load force. Therefore the sprag cam has the self-lock function at a certain rotation angle in which no torque from the rotary actuator is required to maintain its angle. As shown in Fig.4, the self-lock 
condition is satisfied when the leg of the perpendicular line drawn from the center axis of the rotary actuator to the surface of the intermediate cam retainer exists within the contact surface between the retainer and the sprag cam.

\section{Hydraulic Circuit for Rotary Actuator}

The schematic view of the internal structure of the hydraulic rotary actuator is depicted in Fig.5. The chambers a1 and a2, b1 and b2 are connected each other by the conduit passing through the center of the rotor shaft. It has a port A, B and C. The port A and B are connected with the chamber a1, b1, respectively. The port $\mathrm{C}$ opens on the lid of the vane housing and the thickness of the vane covers the port $\mathrm{C}$ around the swing angle, $\theta_{\text {middle, }}$ corresponding to a middle valve lift.

Since engine oil is used as working fluid in the hydraulic system of the VVL, the rotary actuator has to work with the minimum operating pressure of about 0.1-0.2MPa.

Sensor-less 3-step swing angle positioning is possible by the operation of the flow direction control valve as follows;

1) Minimum swing angle positioning for high valve lift The oil is supplied to the port B, and is discharged from the port $\mathrm{A}$. The port $\mathrm{C}$ is closed. The swing angle is held with the self-lock of the sprag cam after rotating to the minimum swing angle, $\theta_{\text {min }}$, by the driving torque generated by the pressure difference between the chambers.

2) Middle swing angle positioning for middle valve lift The oil is supplied to both the port A and B, and is discharged from the port $\mathrm{C}$. The rotor finishes swinging when the vane covers the port $\mathrm{C}$ completely. Since the thickness of the vane is wider than the width of port $\mathrm{C}$, it has a dead band for vane positioning at the angle around the port $\mathrm{C}$. The final swing angle depends on the initial swing angle from which the rotor started swinging. Since no torque is generated in the region where the vane and the port $\mathrm{C}$ fully overlap each other, however, the rotor is positioned at the designed swing angle, $\theta_{\text {middle, }}$ in that region by the self-lock torque of the sprag cam.

3) Maximum swing angle positioning for low valve lift The oil is supplied to the port A, and is discharged from the port $\mathrm{B}$. The port $\mathrm{C}$ is closed. Positioning to the maximum swing angle, $\theta_{\max }$, is the reverse process of the minimum swing angle positioning above-mentioned.

\section{Transition in Pivot Shifting}

Since the large reaction force occurs at the pivot of the intermediate cam in the duration of the valve opening, the pivot maintains its position by the self-lock of the sprag cam. However, if that duration starts while the pivot is shifting, the transient behavior appears in the rotary actuator.

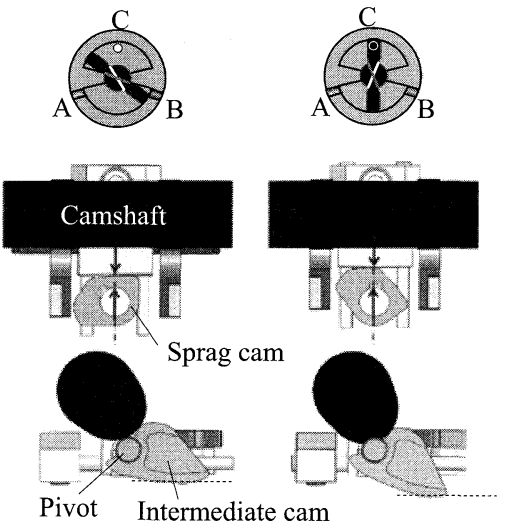

Low valve lift

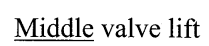

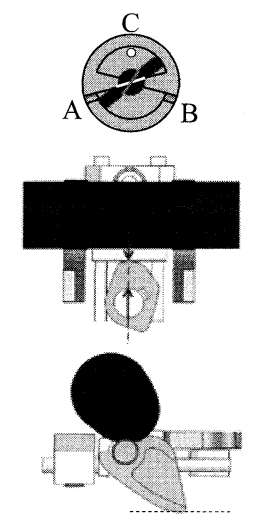

$\underline{\text { High valve lift }}$
Figure 3 Relation between the swing angle of the rotary actuator, the pivot position of the intermediate cam and the valve lift.

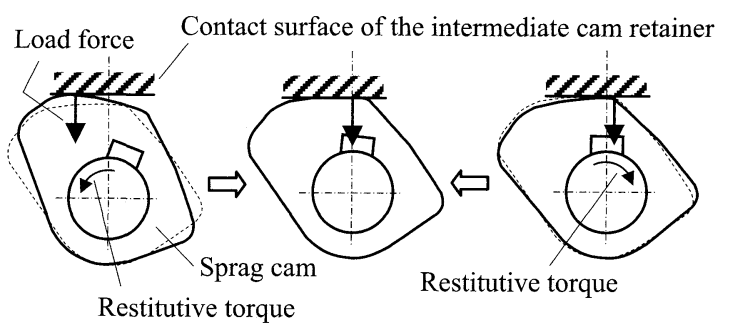

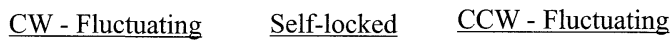

Figure 4 Self-lock function of the sprag cam

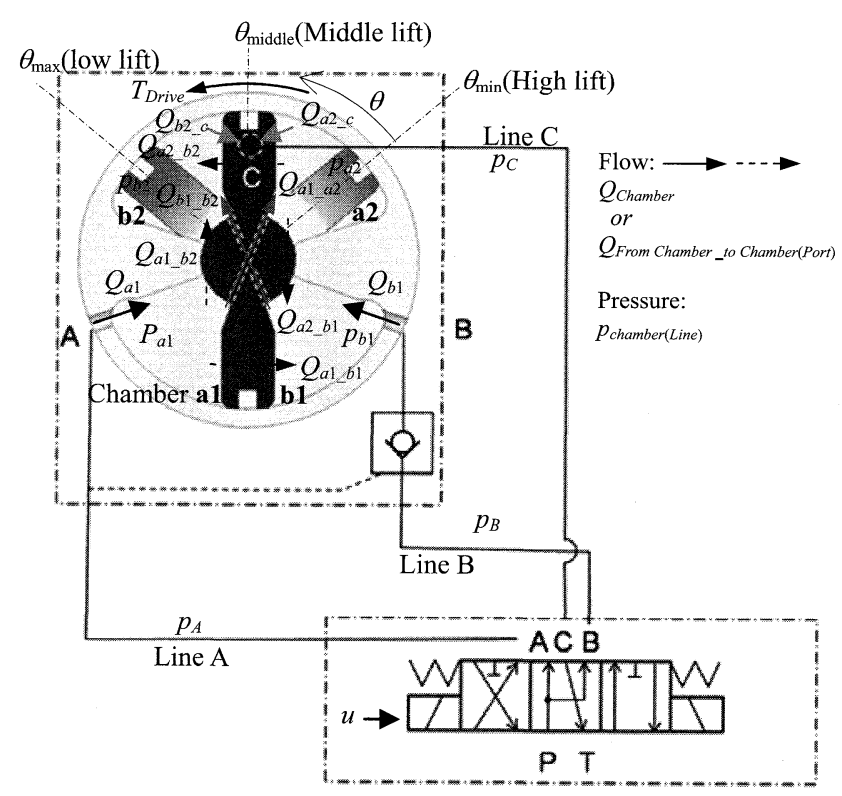

Figure 5 Hydraulic circuit of 3-step rotary actuator

In case that the duration of the valve opening starts while the pivot of the intermediate cam is shifting to the position corresponding to lower valve lift, the rotor 
rotates to the angle corresponding to lower valve lift by assistance of the load torque. Consequently, the rotor reaches to the target swing angle, within some cycles of the camshaft rotation.

However, in case that the duration of the valve opening starts while the pivot is shifting to the position corresponding to higher valve lift, the loaded sprag cam generates the load torque to force the rotor to rotate reverse direction. Due to the load torque, the pressure in the chamber b1 and $\mathbf{b 2}$ easily exceeds the supply pressure of the oil pump. For prevention of the rotor from reverse rotation, as shown in Fig.6, a pilot-operated check valve is installed into the line for the port B. This check valve works as a safety lock and keeps the swing angle in the duration of the valve opening. Then, the rotary actuator restarts and advances its rotation to the target angle corresponding to higher valve lift until next duration of the valve opening starts.

\section{SIMULATION FOR 3-STEP VVL SYSTEM}

\section{Purpose of Simulation}

In order to grasp the behavior of the VVL system, modeling for the VVL system and evaluation of its performance are carried out. In the simulation, the following points are investigated;

1) Influence of the leakage through the clearance between the vane and housing, on the performance of the rotary actuator,

2) Transient pressure change in the chambers,

3) The behavior of the VVL system in case that the duration of the valve opening starts while the pivot of the intermediate cam is shifting.

The model of the VVL system is described in block diagram form to apply MATLAB/SIMULINK to the simulation. The schematic view of the simulated model and symbols of the parameters used in the simulation have been already mentioned in Fig.5.

Fundamental Equations for VVL System Model

1) Dynamics of the hydraulic rotary actuator

$$
I_{m} \ddot{\theta}+B_{m} \dot{\theta}+K_{m} \theta=T_{\text {Drive }}+T_{\text {Load }}
$$

where, $I_{m}, B_{m}, K_{m}, \theta, T_{\text {Drive }}$ and $T_{\text {Load }}$ are the inertia of rotating parts of the rotary actuator, viscous friction coefficient, stiffness of rotational limit, drive torque and load torque, respectively. $K_{m}$ expresses the stiffness equivalent to the contact between the vane and wall of both rotational ends with the nonlinear torsion spring characteristics.

The drive torque $T_{\text {Drive }}$ is given by;

$$
T_{\text {Drive }}=K_{T}\left(p_{a 1}-p_{b 1}\right)+K_{T}\left(p_{a 2}-p_{b 2}\right)
$$

where, $K_{T}$ is a coefficient which transforms the pressure difference between the chambers to the rotor drive

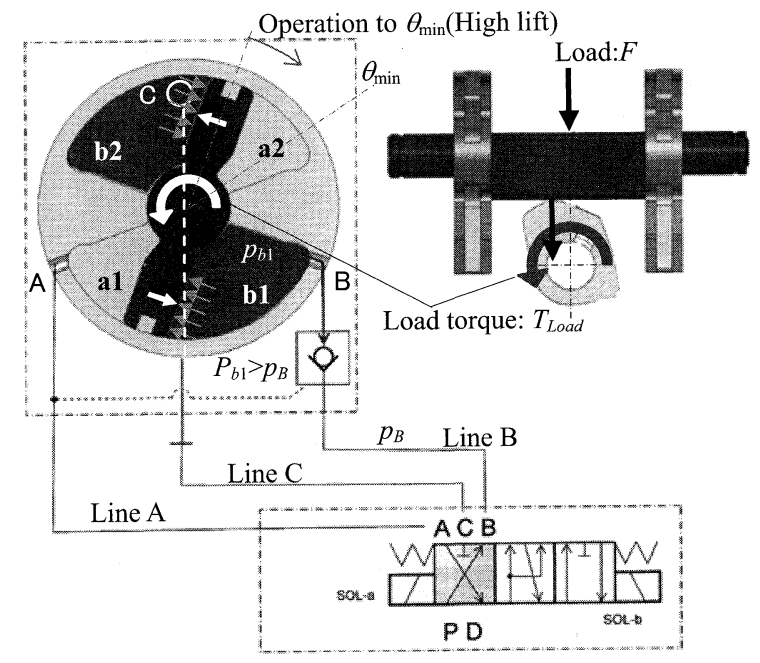

Figure 6 Prevention of the rotor from reverse rotation due to the load torque

(Duration of the valve opening starts while the pivot is sifting to the high valve lift position.)

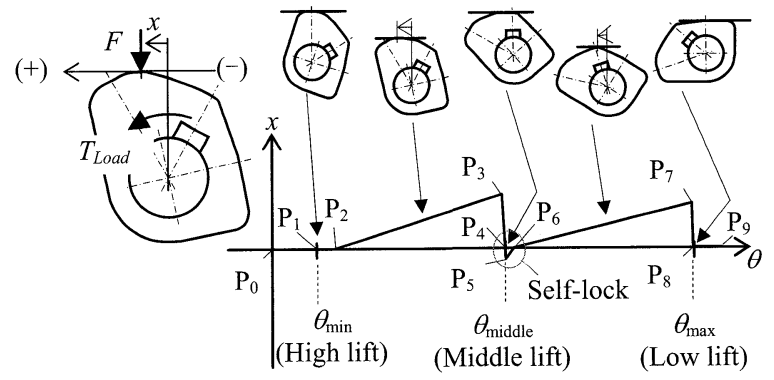

Figure 7 Load torque against every rotor swing angle

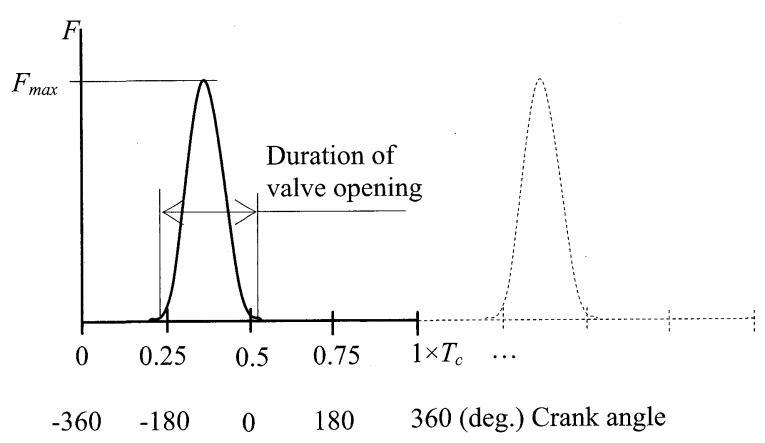

Figure 8 Load force model

( $T_{c}$ : a cycle of crank shaft rotation as angle of $720 \mathrm{deg}$.)

torque, and is derived from the dimensions of vane. Referring to Fig.7, the load torque $T_{\text {Load }}$ is given by;

$$
T_{\text {Load }}=F x
$$

In the simulation, the arm length of the load torque, 


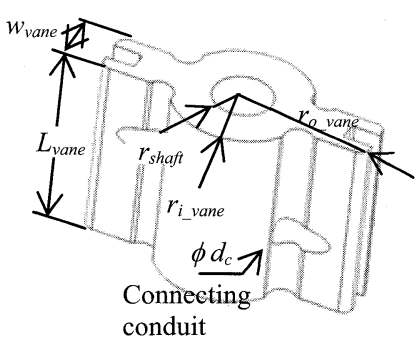

(a)

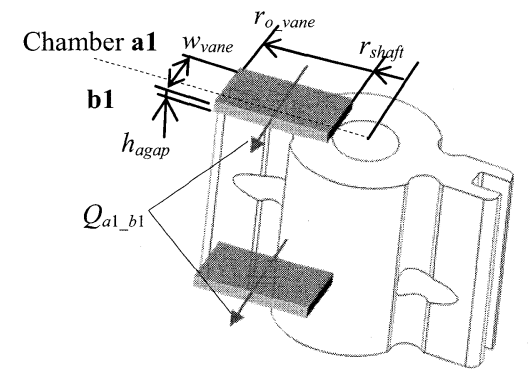

(b)

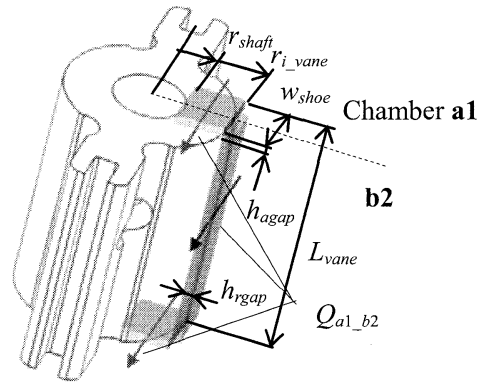

(c)

Dimensions of vane and rotor

Leakage from chamber $\mathbf{a} 1$ to $\mathbf{b} \mathbf{2}$

Figure 9 Model of leakage through the clearances at vane and casing wall

namely the offset $x$ on the sprag cam in Fig.7, is assumed to be given as a function of the rotor swing angle $\theta$ using SIMULINK LOOKUP TABLE, $x=\mathrm{P}(\theta)$, shown in Fig.7.

The load force $F$ acting on the sprag cam is approximated using the form of a periodic function as shown in Fig.8. In Fig.8, $F_{\max }$ is the maximum load force acting on the sprag cam. $T_{c}$ is the period corresponding to a cycle of the crank shaft rotation as angle of $720 \mathrm{deg}$.

2) Continuity at the chamber $\mathbf{a} 1$

$$
\frac{d p_{a 1}}{d t}=\frac{K}{V_{a}}\left(Q_{a 1}-Q_{a 1 \_a 2}-Q_{a 1 \_b 1}-Q_{a 1 \_b 2}-K_{V m} \dot{\theta}\right)
$$

where, $K$ and $V_{a}$ are the bulk modulus of the oil, the volume of the chamber a1, respectively. $K_{V m}$ is the change of the chamber volume against a unit swing angle, which is determined by the dimensions of vane.

Continuity at the chamber $\mathbf{a} \mathbf{2}, \mathbf{b} \mathbf{1}$ and $\mathbf{b} \mathbf{2}$ are formulated in similar form to eq.(4), as well.

3) Flow characteristics at orifice and pipe

The flow characteristics of each flow resistive element, such as a throttle in a valve and lapped area between the port $\mathrm{C}$ and vane width, are approximated using the formula for orifice. The flow characteristics of each piping are approximated using the formula for steady flow through circular pipe.

4) Leakage flow characteristics between chambers

Leakage flow between the chamber a1 and b1 is estimated by the steady flow between stationary and moving flat, parallel walls as shown in Fig.9 (b). The leakage is given by the following formula using the axial clearance $h_{\text {agap }}$;

$$
\begin{aligned}
Q_{a 1 \_b 1}= & 2 \times \frac{\left(r_{o_{-} \text {vane }}-r_{\text {shaft }}\right) h_{\text {agap }}^{3}}{12 \mu w_{\text {vane }}}\left(p_{a 1}-p_{b 1}\right) \\
& +\Delta Q_{a 1 \_b 1}\left(U_{\text {vane }}, p_{a 1}-p_{b 1}\right)
\end{aligned}
$$

Table 1 Design specifications of prototype rotary actuator

\begin{tabular}{|l|l|}
\hline Displacement volume per a chamber & $2.5 \mathrm{~cm}^{3}$ \\
\hline Rotor diameter (outer) $r_{o}$ vane $/$ (inner) $r_{i}$ vane & $28 / 14 \mathrm{~mm}$ \\
\hline Rotor length $L_{\text {vane }}$ & $19 \mathrm{~mm}$ \\
\hline Swing angle & $105 \mathrm{deg}$ \\
\hline Driving torque at supply pressure of $0.1 \mathrm{MPa}$ & $0.25 \mathrm{Nm}$ \\
\hline Response for single step rotation & $0.3 \mathrm{~s}$ \\
\hline Pump Supply pressure (typical)/(minimum) & $0.27 / 0.1 \mathrm{MPa}$ \\
\hline
\end{tabular}

where, $\mu$ is the viscosity of the oil. The first term represents the leakage flow through the stationary flat, parallel walls, the second represent the flow induced by drag of the vane rotation with the circumferential tip speed of the vane, $U_{\text {vane }}$. No leakage is assumed at the tip of vane due to its seal device. Leakage between the chamber $\mathbf{a} \mathbf{2}$ and $\mathbf{b} \mathbf{2}$ is expressed by the same formula as eq. (5), as well.

Leakage between the chamber a1 and b2 is also expressed by the steady flow between stationary and moving flat, parallel walls as shown in Fig.9 (c). Using the axial clearance $h_{\text {agap }}$ and clearance between rotor and shoe $h_{\text {rgap }}$, the leakage is given by;

$$
\begin{aligned}
Q_{a 1 \_b 2}= & \frac{2\left(r_{\text {i_vane }}-r_{\text {shaft }}\right) h_{\text {agap }}+L_{\text {vane }} h_{\text {rgap }}^{3}}{12 \mu w_{\text {shoe }}}\left(p_{a 1}-p_{b 2}\right) \\
& +\Delta Q_{a 1 \_b 2}\left(U_{\text {vane }} p_{a 1}-p_{b 2}\right)
\end{aligned}
$$

The second term also represents the flow induced by drag of the vane rotation. Leakage between the chamber $\mathbf{a} 2$ and $\mathbf{b} 1$ is expressed by the same formula as eq. (6), as well.

5) Flow characteristics between port $C$ and chambers The flow characteristics at the throttle formed by the port $\mathrm{C}$ and vane width are approximated using the formula for orifice. The relation between the rotor swing angle and the opening area of the port $\mathrm{C}$ is expressed by LOOKUP TABLE in SIMULINK.

6) Response of control valves

The response of control valve is approximated using the 


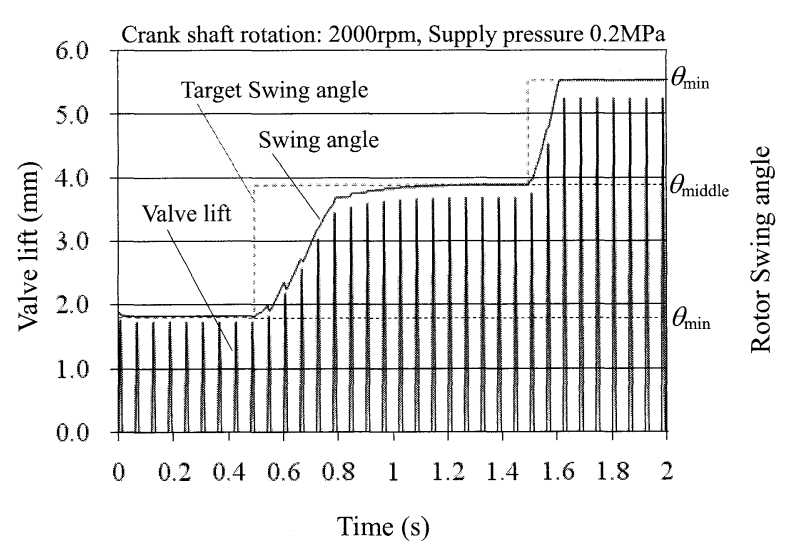

Figure 10 Simulation result for a single step valve lift change from low to middle and from middle to high

first order delay element as a general solenoid valve.

\section{Result of Simulation for 3-Step VVL System}

The design specifications of a prototype VVL is listed in Table 1. One of the examples of the simulated result for the prototype VVL is shown in Fig.10. It indicates that the VVL can change the valve lift within $0.3 \mathrm{~ms}$ at the supply pressure of $0.2 \mathrm{MPa}$ even if it changes the lift higher. In case of the supply pressure of $0.1 \mathrm{MPa}$, it takes $0.5 \mathrm{~s}$ for a single step valve lift change. In case that the axial and radial clearances are $50 \%$ larger than the designed value, the VVL cannot work for the lift change from lower to higher, at the supply pressure of $0.1 \mathrm{MPa}$. Since the leakage reduces the pressure difference between the chambers, it is important to design the clearance for securing the sufficient pressure difference for driving the actuator.

\section{EXPERIMENTAL EVALUATION FOR 3-STEP VVL SYSTEM}

Figure 11 and 12 show the measured performance of the prototype VVL. It is observed that the actuator keeps its swing angle in the duration of valve opening, withstanding the reverse rotation torque in the transition of valve lift change to higher.

The response time is slight longer than the design target, due to the friction of moving parts and the drop of the drive torque by the leakage.

\section{CONCLUSIONS}

This paper has presented the VVL mechanism controlled by the hydraulic 3-step rotary actuator. Simple and reliable VVL system is constituted by a combination of sensor-less drive of the rotary actuator and the specially designed sprag cam. The designed, simulated and measured performances of the developed VVL are listed in Table 2. Optimization of the prototype VVL and development of multi-step mechanism, more than three, with simple structure are starting as a future work.

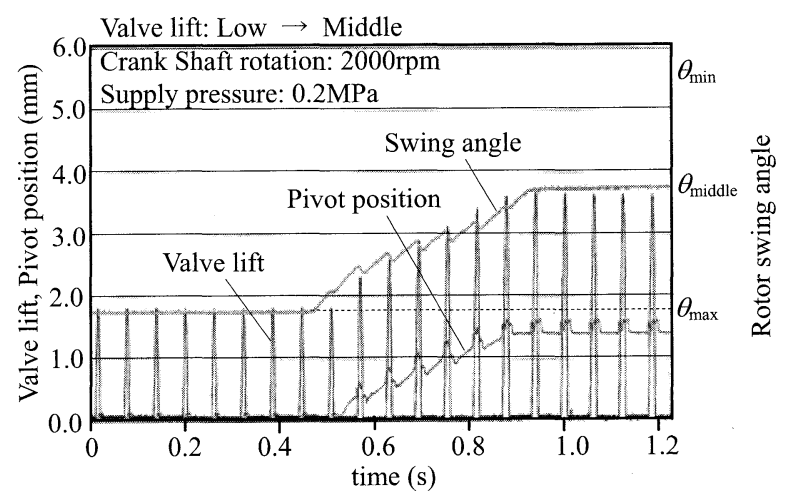

Figure 11 Measured performance of prototype VVL for a step valve lift change from low to middle

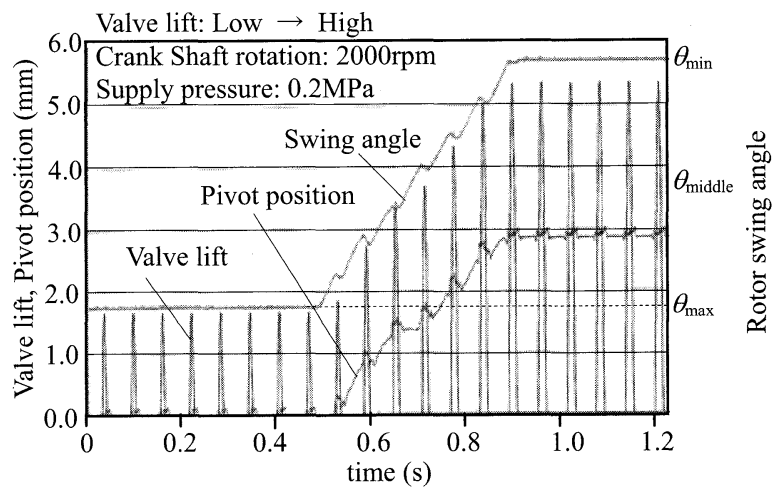

Figure 12 Measured performance of prototype VVL for a step valve lift change from low to high

Table 2 Performance of developed VVL

\begin{tabular}{|l|l|l|l|}
\hline & Design & Simulation & Prototype \\
\hline $\begin{array}{l}\text { Actuator torque at } \\
0.1 \mathrm{MPa}\end{array}$ & $0.25 \mathrm{Nm}$ & $0.25 \mathrm{Nm}$ & $0.25 \mathrm{Nm}$ \\
\hline $\begin{array}{l}\text { Minimum operating } \\
\text { pressure }\end{array}$ & $0.1 \mathrm{MPa}$ & $0.2 \mathrm{MPa}$ & $0.2 \mathrm{MPa}$ \\
\hline Lift holding (Self-lock) & & Good & Good \\
\hline $\begin{array}{l}\text { Response of a single step } \\
\text { lift change }\end{array}$ & $0.3 \mathrm{~s}$ & $0.3 \mathrm{~s}$ & $0.4-0.5 \mathrm{~s}$ \\
\hline
\end{tabular}

\section{REFERENCES}

1. Pierik, R., Burkhard, J., Design and Development of a Mechanical Variable Valve Actuation System, SAE Paper 2000-01-1221, SAE International, 2000

2. Nakamura, M., Hara, S., A Study of a Continuous Variable Valve Event and Lift (VEL) System, SAE Paper 2001-01-243, SAE International, 2001.

3. Flierl, R., Der neue BMW Vierzylinder-Ottomotor mit Valvetronic-Tail1: Konzept und konstruktiver Aufbau (The new BMW Four Cylinder SI Engine with Valvetronic-Part1: Concept, Design and Construction), MTZ, 2001, No.6, pp.450-463.

4. Genise, D., Pierik, R.(Editors), Variable Valve Actuation 2005, SAE Special Publication, SP-1968, SAE International, 2005.

5. Tanaka, H., Toyoda, N., Development of a Sensorless Electrohydraulic Valve Actuator For a Camless Engine, Proceedings of the $6^{\text {th }}$ JFPS International Symposium on Fluid Power TSUKUBA 2005, pp.256-261. 